\title{
Variáveis da primeira fase da integração universitá- ria e mudança de curso
}

\author{
Mudança de curso universitário
}

\author{
António M. Diniz \\ Leandro S. Almeida
}

\begin{abstract}
Resumo
Estudou-se a relação entre variáveis académicas e psicossociais da primeira fase da integração universitária (escolha e colocação num curso) e a Mudança de Curso (MC). As análises bivariadas dos dados de uma amostra de conveniência de 375 estudantes do primeiro ano mostraram que mudaram mais frequentemente de curso aqueles que: tinham Notas de Candidatura (NC) ao Ensino Superior mais baixas; frequentavam cursos ligados a Recursos Tecnológicos (RT); e, atribuíam menor importância aos Determinantes Intrínsecos (DI) na escolha de curso (competências e interesses). Para operacionalizar esta última variável recorreu-se à Escala de Determinantes da Escolha de Curso (EDEC). Entretanto, as variáveis atrás referidas, bem como as outras duas dimensões da EDEC (Agentes Mediadores e Estrutura Ocupacional), também discriminavam os estudantes de acordo com Género. Presume-se, então, que o Género se relaciona indirectamente com a MC, através da interveniência das variáveis NC, Área de Estudos e DI.

Palavras-chave: ensino superior; mudança de curso; diferenças de géneros.
\end{abstract}

\section{Variables of the first half of college integration and change of major}

\begin{abstract}
This study concerned the relationship between academic and psychosocial variables of the first half of college integration (choice and admission to a major) and the Change of Major (CM). Bivariate analyses of data collected using a convenience sample of 375 freshman students showed that those who had changed major more frequently were those who: had lower Access Grades (AG) to Higher Education; were from majors in the field of Technological Resources (TR); and, gave less importance to Intrinsic Determinants (ID) in the choice of major (skills, interests). The latter variable was measured by the Escala de Determinantes da Escolha de Curso (EDEC) [Choice of Major Determinants Scale]. The variables above, as well as the other two dimensions of EDEC (Mediation Agents and Occupational Structure), also significantly discriminated students by gender. Then, we presume that gender is indirectly related to CM, through the mediation of AG, Field of Studies, and ID.
\end{abstract}

Keywords: higher education; change of major; human sex differences.

\section{Variables de la primera fase de la integración universitaria y cambio de curso}

\section{Resumen}

Se estudió la relación entre variables académicas y psico-sociales de la primera fase de la integración universitaria (elección y colocación en un curso) y el cambio de curso (CC). Los análisis bi-variados de los datos de una muestra de conveniencia de 375 estudiantes del primer año mostraron que cambiaron más frecuentemente de curso aquellos que: tenían notas de examen (NE) de ingreso para la universidad más bajas; frecuentaban cursos relacionados a Recursos Tecnológicos (RT); y atribuían una menor importancia a los Determinantes Intrínsecos (DI) en la elección del curso (competencias e intereses). Para evaluar la última variable se recurrió a la Escala de Determinantes da Escolha de Curso (EDEC). Por otro lado, las variables antes relatadas, así como también las otras dos dimensiones de la EDEC (Agentes Mediadores e Estrutura Ocupacional), también discriminaban a los estudiantes de acuerdo con el género. De esa forma, se presume que el género se relaciona indirectamente con la MC por medio de la intervención de las variables NC, Área de Estudios y DI. Palabras clave: enseñanza superior; cambio de curso; diferencias de géneros. 
A influência de factores pessoais e contextuais na adaptação dos caloiros à instituição/curso de acolhimento tem sido analisada face a indicadores de (in)sucesso académico, como o rendimento e a desistência dos estudos. Encontramos estes critérios, principalmente o da desistência dos estudos, comummente referidos desde há muito tempo na literatura anglo-saxónica (e.g., Astin, 1997; Brawer, 1973; Cabrera, Nora, \& Castañeda, 1993; Eaton \& Bean, 1993; Murtaugh, Burns, \& Schuster, 1999; Tinto, 1993). Também os encontramos em vários estudos recentes realizados em Portugal, principalmente voltados para o rendimento académico (Almeida e cols., 2004; Diniz, 200I, 2007; Diniz \& Pinto, 2005; Soares, Almeida, Diniz, \& Guisande, 2006a; Soares, Guisande, Diniz, \& Almeida, 2006b). A hegemonia destes indicadores, justificável pela importância que assumem para os estudantes e suas famílias, e também como indicadores de eficácia institucional (Diniz, 2005), tem mitigado a relevância que a Mudança de Curso (MC) pode ter para a investigação neste domínio. Ora, esta situação não é razoável se pensarmos que a $M C$ pode constituir-se como um critério de adaptação relevante, traduzindo iniciativa na procura de novas soluções face a mudanças vocacionais ou a uma situação académica não desejada, e resultando em permanência no Ensino Superior (ES). Parece, pois, relevante a exploração de factores de caracterização académica e psicossocial dos estudantes que possam estar relacionados com este fenómeno.

Centrando-nos sobre a primeira fase da transição para o ES, a qual compreende a escolha e colocação num curso, devemos considerar a influência que a estrutura sócio-ocupacional exerce sobre a evolução das características, estatutos, escolhas e papéis do indivíduo. Derivando de uma abordagem macro para uma outra mais micro de descrição dessa influência, sabemos que, em função da origem social e do género, o sistema reprodutivo da sociedade condiciona a escolha e a probabilidade de acesso ao ES e mesmo a escolha de determinados cursos (Balsa, Simões, Nunes, \& Campos, 200I; Bourdieu \& Passeron, 1964, 198I; Cabrito, 200I). Os sujeitos consideram menos as escolhas que saem fora do seu habitus (vd. descrição analítica do conceito em Bourdieu, 1989), sugerindo a pressão das normas sociais nas opções individuais (Merton, 1968).

Também sabemos, da microssociologia do trabaIho e das carreiras (Barley, 1989), que as histórias de vida remetem para um pano de fundo social e uma sub-cultura (familiar, comunitária) necessários para perspectivar o desempenho de diferentes papéis individuais. As trajectórias de vida emergem em cenários colectivos contingentes que condicionam os recursos interpretativos individuais, mormente, os significados atribuídos à carreira.

Entretanto, na psicologia vocacional as significações sociais e os contextos relacionais são tidos como informadores do auto-conceito e expectativas, condicionando as aspirações ocupacionais individuais (Gottfredson, 198I, 1996). Com efeito, desde Super (1953) que a escolha ocupacional passou a ser analisada na interacção dinâmica do indivíduo em mudança (dimensão temporal) com um contexto mutável (dimensão sócio-ocupacional). É no seio desta dinâmica que encontramos agentes mediadores da tomada de decisão de carreira, quer de relacionamento íntimo quer de relacionamento institucional. Eles cumprem a função de elos de suporte transcénico (Bronfenbrenner, 1979). Mesmo que não intencionalmente, pais e professores, enquanto modelos profissionais, veiculam estereótipos, influenciando a representação dos jovens quanto ao trabalho e às profissões.

Devemos, então, considerar três níveis interligados de determinação da escolha ocupacional: (I) individual, (2) interpessoal e (3) sócio-institucional. Estes níveis, para o caso da escolha de curso no ES foram operacionalizados através da Escala de Determinantes da Escolha de Curso (EDEC: Diniz, 2004; Diniz, De Abreu, \& Almeida, 1999). Especificando, para (2) e (3) temos os Determinantes Extrínsecos da escolha (DE), englobando constrangimentos e oportunidades da estrutura ocupacional (escolar e profissional), bem como o papel de agentes mediadores (de relacionamento íntimo ou institucional). Para (I) temos os Determinantes Intrínsecos da escolha (DI), relativos às condições pessoais (competências e interesses) que - próprio pensa ter para fazer essa escolha. Estes determinantes poderão relacionar-se com a $M C$, na 
medida em que eles estarão na base de um processo de escolha de curso mais ou menos decidido.

No que concerne às variáveis de caracterização académica dos estudantes, parece admissível considerar-se que o género estará ligado à MC. Ainda que diferenças masculino/feminino não tenham sido confirmadas nalguns estudos sobre (in)decisão vocacional (Crites, 1969; Da Silva, 1997; Gianakos \& Subich, 1986), outros houve em que tais diferenças se verificaram (Lewis \& Savickas, 1995; Lobato \& Koller, 2003; Osipow \& Winer, 1996).

Também a Nota de Candidatura (NC) ao ES poderá estar relacionada com a MC, na medida em que essa nota traduz mais do que um simples número, indicando até que ponto os estudantes trazem consigo as "ferramentas" (e.g., hábitos de estudo, gestão do tempo, cultura académica) necessárias para o sucesso académico. Estudos demonstram que os estudantes com bons resultados no Ensino Secundário têm uma elevada probabilidade de alcançar também bons resultados no ES (Almeida e cols., 2004; Astin, 1997; Diniz, 2007; Diniz \& Pinto, 2005; Marques \& Miranda, 1996; Murtaugh et al., 1999; Soares et al., 2006a,b; Tinto, 1993), o que, putativamente, contribui para a permanência nos cursos.

Acresce, ainda, que a existência de numerus clausus no sistema português de acesso ao ES faz com que certos estudantes ingressem em cursos não desejados (Diniz, 2005). A relação entre número de vagas por Curso/Instituição e a NC condiciona, então, a colocação no Curso da Primeira Escolha (CPE) e, consequentemente, numa dada Área de Estudos (AE). Note-se que mais de um quarto dos estudantes (entre 25 a $30 \%$ ) que se encontram a frequentar o ES português não ficaram colocados nas instituições e cursos mais desejados, parecendo existir uma maior incidência destes casos nos cursos de ciências e tecnologias (Almeida e cols., 2004). Tavares, Santiago e Lencastre (1998), num estudo envolvendo várias universidades portuguesas, constataram que cerca de $40 \%$ dos estudantes de Engenharias não ingressaram no CPE.

Com o presente estudo pretende-se testar as hipóteses que atrás se explanaram e que, muito sucintamente, agora são recordadas. Admite-se, então, a relação entre certas variáveis da primeira fase da integração universitária e a Mudança de Curso. São elas o Género, a Nota de Candidatura, a Área de Estudos, o Curso de Primeira Escolha e as relativas aos Determinantes da Escolha de Curso (DEC): Intrínsecos e Extrínsecos (Agentes Mediadores e Estrutura Ocupacional).

Também se pretende, subsidiariamente, dar particular atenção aos DEC, na medida em que, para além de poderem evidenciar diferenças entre os estudantes que mudaram e os que não mudaram de curso, poderão evidenciar diferenças entre os géneros. Esta última hipótese assenta nas descrições feitas no âmbito da psicologia do desenvolvimento sobre as particularidades de cada um dos géneros nos jovens adultos, nomeadamente, no feminino, a notável saliência das relações sociais e da intimidade na sua vida e, no masculino, a também notável saliência dos objectivos de carreira para a organização da sua vida (Gilligan, 1997; Levinson, 1978).

\section{Método}

\section{Participantes}

Colaboraram voluntariamente no estudo (amostragem de conveniência) 352 estudantes tradicionais (Pascarella \& Terenzini, 1991) (leque etário = 17-24 anos; $M=18$ ). Os estudantes frequentavam pela primeira vez o primeiro ano da Universidade do Minho e estavam distribuídos por cursos ligados a Recursos Humanos (RH: Formação de Professores/ Educadores, Psicologia e Comunicação Social, $n=195$ ) e por cursos ligados a Recursos Tecnológicos (RT: Engenharias e Ciências Exactas Aplicadas, e.g., Química - Ramo Têxtil e Física Aplicada - Ramo Óptica, $n=157)$.

\section{Instrumento}

Para operacionalizar os três níveis de determinação da escolha de curso recorreu-se à Escala de Determinantes da Escolha de Curso (EDEC: Diniz, 2004; Diniz e cols., 1999). Trata-se de uma escala constituída por 12 itens para ordenar por grau decrescente de importância ( $I$ = mais importante), os 
quais estão organizados em torno de três dimensões (cada uma com quatro itens): (I) crença na importância de Determinantes Intrínsecos (DI) para fazer a escolha (e.g., item 7, "Interesse/gosto pela área profissional"; item II, "Maneira de ser e características pessoais"); (2) crença na importância de Determinantes ligados à influência de Agentes Mediadores (DAM) para fazer a escolha (e.g., item 2, "Pais, irmãos ou outros familiares"; item 6, "Professores"); e, (3) crença na importância de Determinantes ligados à Estrutura Ocupacional (DEO) para fazer a escolha (e.g., item I, "Fácil colocação profissional”; item 10, "Número de vagas e médias de ingresso no Ensino Superior").

Visando o teste da validade de constructo da EDEC, recorreu-se ao PREFerenceMAPping2 (Chang \& Carroll, 1972) para tratar os dados recolhidos junto de uma amostra de 370 estudantes universitários do primeiro ano. Este programa baseia-se no modelo do desdobramento (unfolding) criado por Coombs (1950) para o tratamento de dados ordinais de preferência, o qual foi ampliado de unidimensional para multidimensional por Bennett e Hays (in Carrol, 1972) e, depois, refinado por Carroll (1972). Parafraseando Borg e Groenen (1997), com a técnica do desdobramento, assume-se que os estudantes percebem da mesma maneira os 12 itens, mas diferem entre si quanto ao que consideram ser a combinação ideal dos seus atributos. Foi feita a análise interna do modelo (Carroll, 1972; Chang \& Carroll, 1972) tridimensional definido pela EDEC, isto é, partindo das ordenações dos estímulos (escalas I), chegouse às suas coordenadas (escala J). Verificou-se que o modelo hipotético estava bem ajustado aos dados empíricos: $R=0.96$, para o modelo em que os sujeitos atribuem diferentes pesos às dimensões, incluindo a rotação ortogonal dos eixos que as definem (sobre os modelos gerados pelo PREFMAP2, vd. Carroll, 1972).

\section{Procedimento}

A administração da EDEC decorreu em sala de aula, dispensando os professores a parte final do seu tempo lectivo para o efeito. A aplicação ocorreu antes dos participantes no estudo completarem os seus primeiros dois meses de frequência universitária, por forma a captar com maior sensibilidade a realidade em análise, uma vez que estudos indicam que a maior parte das desistências dos cursos ocorre durante o primeiro semestre (Levitz \& Noel, 1989; Myers, 198I; Tinto, 1993). As notas das dimensões resultaram do somatório dos resultados obtidos nos respectivos itens (dado o sentido da ordenação, notas mais elevadas reflectem menor importância). No protocolo de recolha de dados também se colheram informações sobre o Género (masculino= I; feminino=2) e a $A E$ (cursos de $R H=I$; cursos de $R T=2$ ). Recorreu-se, ainda, à informação fornecida pelos Serviços Académicos da Universidade sobre a NC dos estudantes, a colocação no CPE $(\operatorname{sim}=I$; não $=2)$, a $M C(\operatorname{sim}=I$; não $=2)$ e se a $M C$ havia sido para outra $\operatorname{AE}(\operatorname{sim}=1 ;$ não $=2)$.

O tratamento dos dados foi realizado através do SPSS 14.0 for Windows (os valores omissos tiveram um tratamento listwise teste a teste), recorrendo a estatísticas descritivas univariadas (ocorrências, médias de ordenações, médias e desvios-padrão) e estatísticas inferenciais bivariadas, paramétricas ( $t$-Student) e não-paramétricas ( $\chi_{\text {ass. }}^{2}$ e U-Mann-Whitney).

\section{Resultados}

Centrando-nos sobre a MC, primeiro testámos eventuais diferenças nas variáveis de caracterização académica e psicossociológica na amostra, completando este estudo através de uma abordagem segundo o Género e de uma outra, mais fina, considerando cada um dos géneros e a MC.

Assim, na Tabela I apresentam-se as estatísticas descritivas das variáveis de caracterização académica e psicossociológica da amostra, bem como as estatísticas inferenciais resultantes das comparações entre estudantes que mudaram e não mudaram de curso.

Como se constata na tabela, revelaram-se estatisticamente significativas quatro das sete relações testadas. Por comparação com os estudantes que não mudaram de curso, mudaram de curso os estudantes com 
piores NC, que frequentavam mais a $\mathrm{AE}$ de $\mathrm{RT}$ e que atribuíam menor importância aos DI e maior importância aos DEO na sua escolha de curso (lembre-se que na EDEC notas mais elevadas significam menor importância). Destas quatro variáveis, a $A E$ e a DEO foram as que apresentaram menor nível de significância estatística $(p=0.028$ para a AE e $p=0.049$ para a
DEO), o que justifica especial prudência na inferência atrás feita a propósito desta última variável.

Entretanto, na comparação dos resultados obtidos nas variáveis de caracterização académica e psicossociológica da amostra em função do Género (Tabela 2), verificou-se que somente o CPE não diferenciava os estudantes.

Tabela I. Caracterização academica e psicossociológica da amostra em função da Mudança de Curso

\begin{tabular}{|c|c|c|c|c|}
\hline \multirow{2}{*}{ Características } & & \multicolumn{2}{|c|}{ Mudança de Curso } & \multirow[b]{2}{*}{ Comparações $^{\mathrm{a}}$} \\
\hline & & \multirow{3}{*}{$\begin{array}{c}\begin{array}{c}\text { Sim } \\
(n=47)\end{array} \\
33 \\
14\end{array}$} & \multirow{3}{*}{$\begin{array}{c}\begin{array}{c}\text { Não } \\
(N=305)\end{array} \\
202 \\
103\end{array}$} & \\
\hline \multirow[t]{2}{*}{ Género } & Feminino & & & \multirow{2}{*}{$\chi^{2}(1)=.29$} \\
\hline & Masculino & & & \\
\hline \multicolumn{2}{|l|}{ Nota de Candidatura $(\mathrm{NC})^{\mathrm{b}}$} & $\begin{array}{l}115.33 \\
(23.70)\end{array}$ & $\begin{array}{l}136.04 \\
(24.28)\end{array}$ & $t(290)=-4.44^{* * * *}$ \\
\hline \multirow{2}{*}{ Área de Estudos (AE) } & Recursos Humanos (RH) & 19 & 176 & \multirow{2}{*}{$\chi^{2}(1)=4.92^{*}$} \\
\hline & Recursos Tecnológicos (RT) & 28 & 129 & \\
\hline \multirow{2}{*}{$\begin{array}{l}\text { Curso de Primeira Escolha } \\
\text { (CPE) }\end{array}$} & Sim & 9 & 18 & \multirow{2}{*}{$\chi^{2}(1)=3.11$} \\
\hline & Não & 132 & 126 & \\
\hline \multicolumn{2}{|c|}{ Determinantes Intrínsecos $(\mathrm{DI})^{\mathrm{c}}$} & 229.53 & 168.33 & $z=3.86^{* * * * *}$ \\
\hline \multicolumn{2}{|c|}{ Determinantes ligados a Agentes Mediadores (DAM) } & 158.95 & 179.20 & $z=1.27$ \\
\hline \multicolumn{2}{|c|}{ Determinantes ligados à Estrutura Ocupacional $(\mathrm{DEO})^{\mathrm{c}}$} & 149.37 & 180.68 & $z=1.97^{*}$ \\
\hline
\end{tabular}

Tabela 2. Caracterização academica e psicossociológica da amostra em função do Género

\begin{tabular}{|c|c|c|c|c|}
\hline \multirow{2}{*}{ Características } & & \multicolumn{2}{|l|}{ Género } & \multirow[b]{2}{*}{ Comparações ${ }^{\mathrm{a}}$} \\
\hline & & \multirow{2}{*}{ 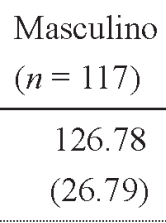 } & $\begin{array}{l}\text { Feminino } \\
(n=235)\end{array}$ & \\
\hline \multicolumn{2}{|l|}{ Nota de Candidatura $(\mathrm{NC})^{\mathrm{b}}$} & & 137.57 & $t(290)=-3.56^{* * * *}$ \\
\hline \multirow{3}{*}{ Área de Estudos (AE) } & & & & \multirow{3}{*}{$\chi^{2}(1)=52.45^{* * * *}$} \\
\hline & Recursos Humanos (RH) & 33 & 162 & \\
\hline & Recursos Tecnológicos (RT) & 84 & 73 & \\
\hline \multirow{2}{*}{$\begin{array}{l}\text { Curso de Primeira Escolha } \\
\text { (CPE) }\end{array}$} & Sim & 47 & 94 & \multirow[b]{2}{*}{$\chi^{2}(1)=0.14$} \\
\hline & Não & 51 & 93 & \\
\hline \multicolumn{2}{|c|}{ Determinantes Intrínsecos $(\mathrm{DI})^{\mathrm{c}}$} & 192.73 & 168.42 & $z=2.12^{*}$ \\
\hline \multicolumn{2}{|c|}{ Determinantes ligados a Agentes Mediadores (DAM) $^{\mathrm{c}}$} & 208.55 & 160.54 & $z=4.18^{* * * *}$ \\
\hline \multicolumn{2}{|c|}{ Determinantes ligados à Estrutura Ocupacional $(\mathrm{DEO})^{\mathrm{c}}$} & 138.45 & 195.44 & $z=4.96^{* * * * *}$ \\
\hline
\end{tabular}

a Entre os géneros. ${ }^{\mathrm{b}}$ Médias (desvios-padrão). ${ }^{\mathrm{c}}$ Médias das ordenações. Teste $t$ assumindo igualdade de variâncias. ${ }^{*} \mathrm{p}<0.05 ;{ }^{* * * *} \mathrm{p}<0.0001$. 
Quanto às restantes variáveis, quando comparados com os estudantes de género feminino, os de género masculino tinham piores NC, frequentavam mais a $A E$ de $R T$, atribuíam menor importância aos DI e aos DAM e maior importância aos DEO. Destas variáveis a DI foi a que apresentou menor nível de significância estatística $(p=0.034)$. Note-se que, salvo as variáveis DAM e DEO, todas as outras são aquelas que se relacionavam de forma estatisticamente significativa com a MC. mudaram de curso, mudaram de curso as estudantes com piores NC, que frequentavam mais a $A E$ de $R T$ e que atribuíam menor importância aos DI.

Para os estudantes do género masculino, verificouse que somente a variável DI apresentou resultados estatisticamente significativos (Tabela 4): quando comparados com os estudantes que não mudaram de curso, mudaram de curso os que atribuíam menor importância aos DI.

Tabela 3. Caracterização académica e psicossociológica dos estudantes do género feminino em função da Mudança de Curso

\begin{tabular}{|c|c|c|c|c|}
\hline \multirow{2}{*}{ Características } & & \multicolumn{2}{|c|}{ Mudança de Curso } & \multirow[b]{2}{*}{ Comparações ${ }^{\mathrm{a}}$} \\
\hline & & \multirow{2}{*}{$\begin{array}{c}\text { Sim } \\
(n=33)\end{array}$} & $\begin{array}{c}\text { Não } \\
(n=202)\end{array}$ & \\
\hline \multicolumn{2}{|l|}{ Nota de Candidatura $(\mathrm{NC})^{\mathrm{b}}$} & & $\begin{array}{l}140.17 \\
(21.65)\end{array}$ & $t(191)=-4.68^{* * * * *}$ \\
\hline \multirow[t]{2}{*}{ Área de Estudos (AE) } & Recursos Humanos (RH) & 16 & 146 & \multirow{2}{*}{$\chi^{2}(1)=7.50^{* *}$} \\
\hline & Recursos Tecnológicos (RT) & 17 & 56 & \\
\hline \multirow{2}{*}{$\begin{array}{l}\text { Curso de Primeira Escolha } \\
\text { (CPE) }\end{array}$} & Sim & 5 & 89 & \multirow{2}{*}{$\chi^{2}(1)=4.03$} \\
\hline & Não & 13 & 80 & \\
\hline \multicolumn{2}{|c|}{ Determinantes Intrínsecos $(\mathrm{DI})^{c}$} & 152.02 & 112.44 & $z=3.12^{* * *}$ \\
\hline \multicolumn{2}{|c|}{ Determinantes ligados a Agentes Mediadores (DAM) ${ }^{\circ}$} & 111.86 & 119.00 & $z=0.56$ \\
\hline \multicolumn{2}{|c|}{ Determinantes ligados à Estrutura Ocupacional $(\mathrm{DEO})^{\mathrm{c}}$} & 99.73 & 120.99 & $z=1.67$ \\
\hline
\end{tabular}

Note-se, ainda, que os resultados obtidos com as variáveis DI, DAM e DEO, demonstram a validade concorrente das dimensões da EDEC face ao critério Género dos estudantes.

Considerando agora os resultados obtidos nas variáveis de caracterização académica e psicossociológica para cada um dos géneros, podemos observar, na Tabela 3, os resultados obtidos para os estudantes do género feminino.

$\mathrm{Na}$ tabela encontramos três das seis relações testadas com resultados estatisticamente significativos: quando comparadas com as que não
Do conjunto dos resultados apresentados nas Tabelas I e 3 e 4 destacam-se a NC, a frequência de uma dada $A E$ e a importância atribuída aos DI, enquanto variáveis que melhor diferenciam os estudantes quanto à MC. Existe, contudo, a nuance de nos estudantes do género masculino somente os DI diferenciarem aqueles que mudaram de curso face aos que o não fizeram. Por último, o ocorrido com a variável DI, tanto para a amostra como para cada um dos géneros, demonstram a validade preditiva desta dimensão da EDEC face ao critério MC. 
Tabela 4. Caracterização académica e psicossociológica dos estudantes do género masculino em função da Mudança de Curso

\begin{tabular}{|c|c|c|c|c|}
\hline \multirow{2}{*}{ Características } & & \multicolumn{2}{|c|}{ Mudança de Curso } & \multirow[b]{2}{*}{ Comparações ${ }^{\mathrm{a}}$} \\
\hline & & $\begin{array}{c}\operatorname{Sim} \\
(n=14)\end{array}$ & $\begin{array}{c}\text { Não } \\
(n=103)\end{array}$ & \\
\hline \multicolumn{2}{|l|}{ Nota de Candidatura $(\mathrm{NC})^{\mathrm{b}}$} & $\begin{array}{l}113.06 \\
(20.49)\end{array}$ & $\begin{array}{l}128.15 \\
(27.05)\end{array}$ & $t(97)=-1.63$ \\
\hline Área de Estudos (AE) & $\begin{array}{l}\text { Recursos Humanos (RH) } \\
\text { Recursos Tecnológicos (RT) }\end{array}$ & $\begin{array}{c}3 \\
30\end{array}$ & $\begin{array}{l}30 \\
73\end{array}$ & $\chi_{(1)}^{2}=0.36$ \\
\hline $\begin{array}{l}\text { Curso de Primeira Escolha } \\
\text { (CPE) }\end{array}$ & $\begin{array}{l}\text { Sim } \\
\text { Não }\end{array}$ & $\begin{array}{l}4 \\
5\end{array}$ & $\begin{array}{l}43 \\
46\end{array}$ & $\chi_{(1)}^{2}=0.05$ \\
\hline \multicolumn{2}{|c|}{ Determinantes Intrínsecos (DI) ${ }^{c}$} & 80.18 & 56.12 & $z=2.50^{*}$ \\
\hline \multicolumn{2}{|c|}{ Determinantes ligados a Agentes Mediadores (DAM) ${ }^{c}$} & 45.82 & 60.79 & $z=1.56$ \\
\hline \multicolumn{2}{|c|}{ Determinantes ligados à Estrutura Ocupacional $(\mathrm{DEO})^{\mathrm{c}}$} & 49.50 & 60.29 & $z=1.12$ \\
\hline
\end{tabular}

a Entre os que mudaram e não mudaram de curso. ${ }^{\mathrm{b}}$ Médias (desvios-padrão). c Médias das ordenações. Teste $t$ assumindo igualdade de variâncias. * $\mathrm{p}<0.05$.

\section{Discussão}

Os resultados deste estudo mostraram que, entre os estudantes tradicionais que pela primeira vez frequentavam o primeiro ano da Universidade do Minho, mudaram mais frequentemente de curso aqueles que: (I) possuíam piores Notas de Candidatura (NC), o que vai de encontro à importância desta variável para, no sistema de acesso ao Ensino Superior (ES) português, os estudantes ingressarem em cursos desejados (Diniz, 2005; Marques \& Miranda, 1996) e para que, já no ES, alcancem bons resultados académicos (Almeida e cols., 2004; Astin, 1997; Diniz, 2007; Diniz \& Pinto, 2005; Marques \& Miranda, 1996; Murtaugh e cols., 1999; Soares e cols., 2006a,b; Tinto, 1993); (2) frequentavam cursos ligados a Recursos Tecnológicos (RT), nos quais parece existir uma maior incidência de ingressos em cursos menos desejados (Almeida e cols., 2004; Tavares e cols., 1998); e, (3) atribuíram menor importância aos Determinantes Intrínsecos (DI) e maior importância aos Determinantes ligados à Estrutura Ocupacional (DEO) para a escolha de curso. Contudo, a afirmação quanto aos DEO deve ser considerada com a maior prudência em razão da significância estatística do resultado obtido, a qual está muito pró- xima do limiar de rejeição. Já quanto aos DI, o resultado encontrado remete para uma menor congruência nos estudantes que mudaram de curso entre as condições pessoais (competências e interesses) associadas à sua escolha de curso e a sua colocação num dado curso. Este resultado revela a capacidade desta dimensão da Escala de Determinantes da Escolha de Curso (EDEC: Diniz, 2004; Diniz e cols., 1999) para discriminar grupos conhecidos (estudantes que mudaram versus não mudaram de curso), demonstrando a sua validade preditiva face à Mudança de Curso (MC).

Realça-se, ainda, o facto de, na amostra, a colocação no Curso de Primeira Escolha (CPE) não se ter relacionado com a MC, o que vai de encontro a estudos com amostras idênticas à nossa (Soares e cols., 2006 a,b). Através do recurso à análise de trajectórias (path analysis), neles verificou-se a inexistência de qualquer efeito (directo ou indirecto) da variável CPE sobre o grau de investimento cognitivo (expectativas de envolvimento académico) e comportamental (envolvimento) dedicados pelos estudantes à vida académica, o grau de satisfação académica e o rendimento académico. Também se verificou no presente estudo que o Género não estava relacionado com a $M C$, o que estará ligado à indiferenciação entre masculino e feminino encontrada por outros autores quan- 
to ao fenómeno da (in)decisão vocacional (Crites, 1969; Da Silva, 1997; Gianakos \& Subich, 1986).

Entretanto, na comparação entre os géneros, foram os estudantes do género masculino que: (I) apresentaram piores NC, em consonância com o encontrado por Vasconcelos, Almeida e Monteiro (2005); (2) frequentavam mais os cursos de RT, em consonância com o encontrado em outros estudos com universitários portugueses (Diniz, 200I; Soares e cols., 2006a) e de acordo com o expectável em face da influência do estereótipo social do género na escoIha ocupacional (Gottfredson, 198I, 1996) ou, por outras palavras, da influência do sistema reprodutivo da sociedade (Balsa e cols., 200I; Bourdieu \& Passeron, 1964, 198I; Cabrito, 200I) sobre essa escolha; e, (3) atribuíram menor importância aos DI e aos Determinantes ligados a Agentes Mediadores e maior importância aos DEO para fazer a sua escolha de curso, o que poderá justificar-se em razão da sua maior orientação para a realidade ocupacional, fruto da saliência que tem na organização da sua vida a perseguição de objectivos de carreira (Levinson, 1978). Por outro lado, da maior saliência que o relacionamento interpessoal e a intimidade têm na vida dos sujeitos do género feminino (Gilligan, 1997), terão resultado as diferenças entre os géneros, favoráveis ao feminino nos DI e nos DAM. Estes resultados revelam a capacidade das três dimensões da EDEC (DI, DAM e DEO) para discriminar grupos conhecidos (estudantes do género masculino versus feminino), demonstrando a sua validade concorrente face ao critério Género.

Então, confrontando resultados obtidos na comparação entre os géneros com os obtidos para a MC, ainda que o Género não esteja lhe directamente relacionado, é plausível crer numa relação indirecta entre estas duas variáveis, mediada pela NC, pela frequência de uma dada $A E$ e pela importância atribuída aos DI na escolha de curso. Esta hipótese poderá ser verificada através do teste de um modelo de trajectórias (path analysis) que contemple essas relações indirectas entre Género e MC.

Por fim, convém realçar que os resultados apurados provieram de uma amostra não representativa, com os consequentes problemas de generaliza- ção, mesmo para os estudantes do primeiro ano da Universidade do Minho. Por exemplo, nela não constam os estudantes que faltaram às aulas em que decorreu a recolha de dados, nem os estudantes de outros cursos e de outras áreas de estudos, nem os estudantes que mudaram de curso para outra instituição. Assim, eles devem ser tidos como indicadores relativos aos estudantes que apresentam características académicas semelhantes às da amostra aqui considerada.

\section{Referências}

Almeida, L. S. (Coord.), Gonçalves, A., Soares, A. P., Marques, A. P., Fernandes, E., Machado, C., Casal, M., \& Vasconcelos, R. (2004). Transição, adaptação e rendimento académico de jovens no Ensino Superior (Relatório Final de Projecto). Braga: Universidade do Minho.

Astin, A. W. (1997). What matters in college? Four critical years revisited ( ${ }^{\mathrm{a}}$ ed.). San Francisco, CA: Jossey-Bass.

Barley, S. R. (1989). Careers, identities, and institutions: The legacy of the Chicago School of Sociology. Em M. B. Arthur, D. T. Hall \& B. S. Lawrence (Eds.), Handbook of career theory (pp. 4I-65). Cambridge, MA: Cambridge University Press.

Balsa, C., Simões, J. A., Nunes, P., Carmo, R., \& Campos, R. L. (200I). Perfil dos estudantes do Ensino Superior: Desigualdades e diferenciação. Lisboa: Colibri.

Bourdieu, P. (1989). Langage et pouvoir simbolique. Paris: Seuil.

Bourdieu, P. \& Passeron, J.-C. (1964). Les héritiers: Les étudiants et la culture. Paris: Minuit.

Bourdieu, P., \& Passeron, J.-C. (198I). La reproduccion: Elementos para una teoria del sistema de ensañanza ( $2^{\mathrm{a}}$ ed.). Barcelona: Laia. (Original publicado em 1970).

Brawer, F. B. (1973). New perspectives on personality development in college students. San Francisco, CA: Jossey-Bass.

Bronfenbrenner, U. (1979). The ecology of human development: Experiments by nature and design. Cambridge, MA: Harvard University Press.

Cabrera, A. F., Nora, A., \& Castañeda, M. B. (1993). Stuctural equations modeling test of an integrated model of student retention. Journal of Higher Education, 64(2), I23- 139. 
Cabrito, B. G. (200I). Universidade e representação social. Revista de Educação, I0(2), 25-37.

Carroll, J. D. (1972). Individual differences and multidimensional scaling. Em R. N. Shepard, A. K. Romney \& S. B. Nerlove (Eds.), Multidimensional scaling: Theory and applications in the behavioral sciences (vol. I, pp. 105-I55). New York: Seminar Press.

Chang, J. J., \& Carroll, J. D. (1972). How to use PERFMAP and PREFMAP2: Programs which relate preference data to multidimensional scaling solution. Manuscrito não publicado. Murray Hill, NJ: Bell Telephone Laboratories. [On-line] Disponível: How to use PERFMAP: A program which relates preference data to multidimensional scaling solution, http:// marketing.byu.edu/htmlpages/books/pcmds/PREFMAP.html. Recuperado: jan. 2003.

Coombs, C. H. (1950). Psychological scaling without a unit of measurement. Psychological Review, 57(3), I45-I58.

Crites, J. O. (1969). Vocational psychology. New York: McGrawHill.

Da Silva, J. M. T. (1997). Dimensões da indecisão vocacional: Investigação com adolescentes. Tese de Doutorado, não publicada, Universidade de Coimbra, Coimbra.

Diniz, A. M. (200I). Crenças, escolha de carreira e integração universitária. Tese de Doutorado, não publicada, Universidade do Minho, Braga.

Diniz, A. M. (2004). Escala de Determinantes da Escolha de Curso. Em L. S. Almeida, M. R. Simões, M. M. Gonçalves \& C. Machado (Coords.), Avaliação psicológica: Instrumentos validados para a população portuguesa (vol. 2, pp. 7I-86). Coimbra: Quarteto.

Diniz, A. M. (2005). A Universidade e os seus estudantes: Um enfoque psicológico. Lisboa: ISPA.

Diniz, A. M. (2007, Janeiro). Integração e rendimento escolar dos estudantes universitários do $I^{\circ}$ ano. Comunicação apresentada no Ciclo de Palestras do Departamento de Economia do Instituto de Economia e Gestão (ISEG) da Universidade Técnica de Lisboa, ISEG, Lisboa.

Diniz, A. M., De Abreu, J. G., \& Almeida, L. S. (1999). Escalamento multidimensional de uma escala de dados de preferência. Psychologica, 2I, 20I-2I5.

Diniz, A. M., \& Pinto, M. S. (2005). Integração universitária e rendimento académico. Psicologia e Educação, 4(2), 43-56.

Eaton, S. B., \& Bean, J. P. (1993, November). An approach/ avoidance model of college student retention. Paper presented at the Annual Meeting of the Association for the Study of Higher Education, Pittsburg, PA.

Gianakos, I., \& Subich, L. M. (1986). The relationship of gender and sex-role orientation to vocational undecidness. Journal of Vocational Behavior, 29(I), 42-50.

Gilligan, C. (1997). Teoria psicológica e desenvolvimento da mulher. Lisboa: Fundação Calouste Gulbenkian. (Original publicado em 1982).

Gottfredson, L. S. (198I). Circunscription and compromisse: A development theory of occupational aspirations. Journal of Counseling Psychology (Monograph), 28(6), 545-79.

Gottfredson, L. S. (1996). Gottfredson's theory of circunscription and compromisse. E, D. Brown \& L. Brooks (Eds.), Career choice and development ( $3^{\mathrm{a}}$ ed., pp. 179-232). San Francisco, CA: Jossey-Bass.

Levinson, D. (1978). The seasons of a man's life. New York: Ballantine.

Levitz, R., \& Noel, L. (1989). Connecting students to institutions: Keys to retention and success. Em M. L. Upcraft, J. N. Gardner \& Associates (Eds.), The freshman year experience: Helping students survive and succeed in college (pp. 65-8I). San Francisco, CA: Jossey-Bass.

Lewis, D. M., \& Savickas, M. L. (1995). Validity of the Career Factors Inventory. Journal of Career Assessment, 3(I), 44-56.

Lobato, C. R. P. S., \& Koller, S. H. (2003). Maturidade vocacional e gênero: Adaptação e uso do Inventário Brasileiro de Desenvolvimento Profissional. Revista Brasileira de Desenvolvimento Profissional, 4(I/2), 57-69.

Marques, J. F., \& Miranda, M. J. (1996). Access to Higher Education in Portugal: Selection procedures revisited from studies at the University of Lisbon. Oxford Review of Education, 22(3), 337-347.

Merton, R. K. (1968). Social theory and social structure (3. ed.). Glencoe, IL: The Free Press.

Murtaugh, P. A., Burns, L. D., \& Schuster, J. ( 1999). Predicting the retention of university students. Research in Higher Education, 40(3), 355-37I.

Myers, E. (198I). A comparative analysis of persisters, permanent dropouts, dropouts who transfer and stopouts at St. Cloud State University. Dissertation Abstracts International, 42, I05A.

Osipow, S. H., \& Winer, J. L. (1996). The use of the Career Decision Scale in career assessment. Journal of Career Assessment, 4(2), I I7-130. 
Pascarella, E. T., \& Terenzini, P. T. (1991). How college affects students: Findings and insights from twenty years of research. San Francisco, CA: Jossey-Bass.

Soares, A. P., Guisande, M. A., Diniz, A. M., \& Almeida, L. (2006a). Transición a la Enseñanza Superior: Contrucción y validación de um modelo multidimensional de ajuste de los jóvenes al contexto universitario. Psicothema, I8(2), 249-255.

Soares, A. P., Almeida, L. S., Diniz, A. M., \& Guisande, M. A. (2006b). Modelo multidimensional de ajustamento de jovens ao contexto universitário (MMAU): Estudo com estudantes de ciências e tecnologias versus ciências sociais e humanas. Análise Psicológica, 24(I), I5-28.
Super, D. E. (1953). A theory of vocational development. American Psychologist, 8(I), 185-190.

Tavares, J., Santiago, R. A., \& Lencastre, L. (1998). Insucesso no $I^{\circ}$ ano do ensino superior: Um estudo no âmbito dos cursos de licenciatura em Ciências e Engenharia da Universidade de Aveiro (Relatório de Pesquisa). Aveiro: Universidade de Aveiro.

Tinto, V. (1993). Leaving college: Rethinking the causes and cures of student attrition (2. ed.). Chicago: University of Chicago Press.

Vasconcelos, R. M., Almeida, L. S., \& Monteiro, S. C. (2005). Métodos de estudo em alunos do $1^{\circ}$ ano da universidade. Psicologia Escolar e Educacional, 9(2), 195-202.

Recebido em: 05/10/2007

Revisado em : 05/I2/2007

Aprovado em: 10/02/2008

Sobre os autores

António M. Diniz (antonio.diniz@ispa.pt) - Doutor em Psicologia da Educação pela Universidade do Minho. Prof. Auxiliar no ISPA. Investigação em avaliação psicológica, construção e adaptação de provas psicológicas, métodos quantitativos e qualitativos aplicados ao estudo do jovem adulto universitário e do adulto idoso.

Endereço para correspondência.

ISPA - Instituto Superior de Psicologia Aplicada

Rua Jardim do Tabaco, 34

| |49-04| Lisboa, Portugal

Leandro S. Almeida - Doutor em Psicologia da Educação pela Universidade do Porto. Professor Catedrático do Instituto de Educação e Psicologia da Universidade do Minho. Investiga nas áreas da cognição, aprendizagem, avaliação psicológica, construção e validação de instrumentos. Autor de provas de avaliação cognitiva.

Universidade do Minho, Braga, Portugal 